\title{
Possible role of miR-204 in optic nerve injury through the regulation of GAP-43
}

\author{
NANYE WANG $^{1 *}$, WENYAN YANG ${ }^{2 *}$, TINGTING XIAO $^{1}$, ZHENZHONG MIAO $^{1}$, \\ WENBIN LUO ${ }^{1}$, ZHIPENG YOU ${ }^{1}$ and GUODONG LI ${ }^{1}$ \\ ${ }^{1}$ Department of Ophthalmology, The Second Affiliated Hospital of Nanchang University, \\ Nanchang, Jiangxi 330006; ${ }^{2}$ Department of Ophthalmology, The First Affiliated Hospital of \\ Kunming Medical University, Kunming, Yunnan 650034, P.R. China
}

Received December 15, 2016; Accepted September 6, 2017

DOI: $10.3892 / \mathrm{mmr} .2017 .8341$

\begin{abstract}
Optic nerve injury is a common disease. The present study aimed to examine the possible role of microRNA-204 (miR-204) in optic nerve injury through the regulation of growth-associated protein-43 (GAP-43). Initially, optic nerve injury models were established in Sprague-Dawley (SD) rats, and the function of miR-204 was either enhanced or inhibited through injection of miR-204 mimic and inhibitor, respectively. Subsequently, the mRNA and protein levels of miR-204, GAP-43, toll-like receptor 4 (TLR4), myeloid differentiation factor $88(\mathrm{MyD} 88)$ and nuclear factor- $\kappa \mathrm{B}(\mathrm{NF}-\kappa \mathrm{B})$ were examined in retinal tissues using reverse transcription-quantitative polymerase chain reaction and western blot analyses. The apoptosis of retinal tissue cells was also detected using a terminal deoxynucleotidyl transferase mediated dUTP nick end labeling assay. There was a significant increase in the level of miR-204 in retinal blood vessels of the model SD rats, compared with that in the normal SD rats $(\mathrm{P}<0.05)$, and the expression of GAP-43 was significantly decreased $(\mathrm{P}<0.05)$. The results confirmed that the expression of GAP-43 was significantly reduced, compared with that in the normal control group when the rats were treated with miR-204 mimic $(\mathrm{P}<0.05)$, which was similar to the result in the model group. By contrast, its expression of GAP-43 was significantly increased when treated with the miR-204 inhibitor $(\mathrm{P}<0.05)$. Compared with the normal control group, the expression levels of TLR4, MyD88 and NF- $\mathrm{B}$ were significantly increased in the miR-204 mimic group and model group $(\mathrm{P}<0.05)$, whereas
\end{abstract}

Correspondence to: Dr Guodong Li, Department of Ophthalmology, The Second Affiliated Hospital of Nanchang University, 1 Minde Road, Nanchang, Jiangxi 330006, P.R. China

E-mail: nannjdhw@sina.com

${ }^{*}$ Contributed equally

Key words: microRNA-204, growth-associated protein 43, optic nerve injury, toll-like receptor 4 , myeloid differentiation factor 88 , nuclear factor- $\kappa \mathrm{B}$ the same three factors in the miR-204 inhibitor group were effectively inhibited, compared with those in the model group, and showed similar results to the normal control group. The apoptotic rates of retinal cells in the miR-204 mimic group and model group were significantly increased, compared with that in the normal control group $(\mathrm{P}<0.05)$, whereas miR-204 inhibitor effectively reversed the effects on apoptotic rate observed in the model group, showing similar results to those in the normal control group. Taken together, miR-204 promoted the apoptosis of retinal cells through inhibiting GAP-43, providing theoretical guidance for the function of GAP-43 in retinal injury.

\section{Introduction}

Optic nerve injury results from damage to the optic nerve in craniocerebral trauma, with the majority of cases caused through an indirect mechanism (1). Optic nerve injury is commonly the result of ocular surgical injury, which refers to the complete or partial loss of the optic nerve due to the effect of external force or through direct contact with tools (2). The progressive death of retinal ganglion cells (RGCs) is a major cause of irreversible visual impairment following optic nerve injury. Clinically, there are no effective treatments for recovering visual function at present. However, a previous study examined the Wallerian degeneration of disconnected axonal fragments following secondary axotomy 12 weeks following injury to an optic nerve; the results revealed that a number of nerve fibers initiated Wallerian degeneration within the days and weeks following the initial mechanical injury to the optic nerve (3).

Growth-associated protein-43 (GAP-43) is a cell membrane phosphorylation protein of the nerve terminal membrane, which belongs to the family of calmodulin-binding proteins (4). The protein consists of two functional regions, including a membrane binding area and $G$ protein reaction zone (5). It is mainly distributed in the brain, cerebellum, hippocampus, spinal cord and autonomic nervous system. In general, the levels of GAP-43 in young animals are 20 times higher than those of adult animals, whose organs have developed fully (6). GAP-43 is a neuronal-specific protein, which regulates multiple aspects of neuronal development, plasticity 
and regeneration (7). It is closely associated with nerve growth and synapse formation, particularly in nerve regeneration. It has been shown to promote bone marrow mesenchymal stem cell differentiation in a rat model of traumatic optic neuropathy (8), which may be effective for the treatment of central nervous system disorders (9). Investigation of the time course of the expression of GAP-43 in the retina of goldfish, in which axons are able to regrow for full restoration of visual function following optic nerve transection, demonstrated that GAP-43 was a useful biochemical marker for monitoring the entire period of optic nerve regeneration in the fish (10). Existing evidence also shows that GAP-43 can increase by 20-100 times following peripheral nerve damage $(11,12)$.

Nerve injury usually induces high levels of inflammatory cytokines, and these inflammatory factors lead to neuritis injury (13). The toll-like receptor 4 (TLR4) signaling pathway acts via a myeloid differentiation factor 88 (MyD88)-independent pathway, leading to the subsequent activation of nuclear factor- $\kappa \mathrm{B}(\mathrm{NF}-\kappa \mathrm{B})$ gene binding, which induces a variety of cytokines (14). MyD88 is a key joint molecule of TLR signaling, with an important role in transferring upstream information. MyD88 is a member of the Toll/interleukin-1 receptor (IL-1R) family and the family members of the death domain family (15). Mechanistic experiments have showed that dioscin significantly increases the levels of heat shock protein 70 , decreases the levels of TLR4, MyD88, tumor necrosis factor receptor-associated factor 6 , cyclooxygenase-2, c-Jun N-terminal kinase, extracellular signal-regulated kinase and p38 mitogen-activated protein kinase phosphorylation, suppresses the nuclear translocation of NF- $\mathrm{kB}$ and high mobility group box 1 (HMGB1), and subsequently decreases the mRNA levels of IL- $1 \beta$, IL-6, tumor necrosis factor- $\alpha$, intercellular adhesion molecule- 1 and interferon- $\gamma(16)$. The TLR4-mediated phosphatase and tensin homolog/phosphoinositide 3-kinase/AKT/NF- $\kappa$ B signaling pathway in rat hippocampal neurons is associated with the activation of a neuroinflammatory response (17). Another study showed that TLR4 is critical in streptozotocin-induced diabetic retinopathy at the level of inflammatory cytokine induction, via MyD88-dependent and MyD88-independent pathways (18).

MicroRNAs (miRNAs) are non-coding, single-stranded small RNA molecules, which can inhibit or upregulate the transcription of a target mRNA, or induce its degradation through complete or incomplete pairing with the 3 ' untranslated region ( $3^{\prime}$ UTR) of the target mRNA, consequently regulating the expression of genes (19-21). Studies have shown that miRNA-204 (miR-204) has multiple physiological functions, including that of a master regulator in the regulation of lens morphogenesis in vertebrates, and tumor-suppressor genes in the types of cancer investigated (22); miR-204 regulates the differentiation process of mesenchymal stem cells through decreasing the expression of Runt-related transcription factor 2 in mesenchymal stem cells (23); therefore, downregulating the expression of miR-204 may be a novel strategy for treating aplastic anemia (24). In addition, this miRNA is considered particularly important in regulating eye development, affecting multiple ocular functions (22), as it is indispensable for retinal pigmented epithelium (RPE) differentiation and development of the adjacent neuroretina (25), and is involved in corneal epithelial cell proliferation and migration (26). Studies have indicated that miRNAs are important in the regulation of several neural correlates of the loop in diseases, including brain trauma and cerebral ischemia, the pathogenesis of which may be contributed to by the change of synaptic plasticity $(21,27)$. In addition, investigations have indicated that the expression of miR-204 is high in nerve terminals; however, the mechanism involved in the regulation of visual injury remains to be fully elucidated (28).

The present study aimed to examine the function of miR-204 through modulating the expression of GAP-43 in the repair process following optic nerve injury. A model of optic nerve injury was established in Sprague-Dawley (SD) rats, and the expression levels of miR-204 and GAP-43 were detected in retinal blood vessels of model and normal SD rats. Subsequently, miR-204 mimic or inhibitor was injected into the model SD rats, and reverse transcription-quantitative polymerase chain reaction (RT-qPCR) analysis was performed to detect miR-204 and the mRNA levels of GAP-43. The protein expression levels of GAP-43 TLR4, MyD88 and NF- $\kappa$ B were detected using western blot analysis, and the apoptosis of retinal cells was detected using the terminal deoxynucleotidyl transferase mediated dUTP nick end labeling (TUNEL) method. The results may provide a theoretical basis for the clinical treatment of optic nerve injury.

\section{Materials and methods}

Reagents and equipment. The miR-204 mimic and inhibitor were from Shenggong Biotech Co. (Shanghai, China), for injecting into the cavities of the retina. Primary antibodies against GAP-43, TLR4, MyD88 and NF- $\kappa$ B were from Abcam (Cambridge, UK); the ChemiDoc ${ }^{\mathrm{TM}}$ XRS gel imaging system and fluorescence qPCR instrument were from Bio-Rad Laboratories, Inc. (Hercules, CA, USA); SYBR-Green qPCR SuperMix was from Invitrogen; Thermo Fisher Scientific, Inc. (Waltham, MA, USA); the TUNEL apoptosis kit was from Beyotime Institute of Biotechnology (Haimen, China); the mini double vertical electrophoresis and mini transfer electrophoresis apparatus were from Beijing 61 Instrument Factory (Beijing, China); the inverted fluorescence microscope was from Leica Microsystems, Inc. (Buffalo Grove, IL, USA).

Animals and induction of optic nerve crush injury. A total of 20 male SD rats (8-12 weeks; $150-200 \mathrm{~g}$ ), all of which conformed to the standard of first class animal, were purchased from Hunan Slack King Experimental Animal Co. (Changsha, China). The animals were reared in the isolators under the conditions of a $10-15^{\circ} \mathrm{C}$ temperature, humidity of $55-75 \%$ and a $12 \mathrm{~h}$ light:dark cycle, with free access to food and drinking water. The animals had no external eye disease, and the direct and indirect light reflexes of pupils in both eyes were normal. The 20 SD rats were randomly divided into the following four groups, each group containing 5 rats: Normal control group; model + miR-204 mimic group; model + miR-204 inhibitor group; and model group. The present study was approved by the Ethics Committee of the Department of Ophthalmology, The Second Affiliated Hospital of Nanchang University, Medical School of Nanchang University (Jiangxi, China). 
Animal model. The animal model was established using the following methods: Pentobarbital sodium $(30 \mathrm{mg} / \mathrm{kg})$ was used for anesthesia by intraperitoneal injection, followed by routine disinfection, draping, and subcutaneous injection of lidocaine in the upper eyelid for local anesthesia. A horizontal incision of $1 \mathrm{~cm}$ was made in the region, $3 \mathrm{~mm}$ from the eyelid. The subcutaneous tissue was separated bluntly, entering the eye socket closely along the orbital bone. A section of orbital fat was removed, part of the extraocular muscle was cut, and the optic nerve was carefully exposed, holding the optic nerve for $10 \mathrm{sec}, 2 \mathrm{~mm}$ away from the posterior globe, avoiding damage to the ophthalmic artery. A fundus microscope was used to observe the above process. Those rats in which retinal blood flow was recovered within 5 min following crushing injury were included in the experiment. Following lamination oversewing, erythromycin eye ointment was administered topcially onto the wound and an intramuscular injection of penicillin sodium (1.2 $\mu \mathrm{g}$ daily) was administered postoperatively for 3 days to promote healing.

RNA extraction and fluorescence RT-qPCR analysis. The retinal vascular tissues of the SD rats were used for RNA extraction, following which cDNA was synthesized according to the manufacturer's instructions of the RT kit. This cDNA was used as the template for fluorescence qPCR, using GAPDH as an internal control. The relative expression levels of miR-204, GAP-43, TLR4, MyD88 and NF- $\kappa$ B in each group were calculated.

RNA was extracted from the frozen retina biopsies using the mirVana ${ }^{\mathrm{TM}}$ miRNA isolation kit (Applied Biosystems; Thermo Fisher Scientific, Inc.). RT-qPCR analysis of the miR-204 transcripts was performed using TaqMan ${ }^{\circledR}$ microRNA assays (Applied Biosystems; Thermo Fisher Scientific, Inc.), using RNU48 levels to normalize the data. The RT-qPCR reactions were performed in triplicate in 296-well plates and were run in a QuantStudio ${ }^{\mathrm{TM}} 12 \mathrm{~K}$ Flex Real-Time PCR system. The $2^{-\Delta \Delta C q}$ method (29) was used to determine the relative quantitative levels of miR-204, TLR4, MyD88, NF- $\kappa$ B and GAPDH. RT-qPCR analysis of gene expression was performed using SYBR Green PCR master mix (Applied Biosystems; Thermo Fisher Scientific, Inc.) and the expression of GAPDH was used as an endogenous control for normalization purposes. PCR reaction was performed using the following conditions: Pre-denaturation at $95^{\circ} \mathrm{C}$ for $5 \mathrm{~min}$. denaturalization at $95^{\circ} \mathrm{C}$ for $1 \mathrm{~min}$, annealing at $60^{\circ} \mathrm{C}$ for $45 \mathrm{sec}, 72^{\circ} \mathrm{C}$ for $45 \mathrm{sec}, 35$ cycles of amplification, and final extension at $72^{\circ} \mathrm{C}$ for $10 \mathrm{~min}$. A total of $2 \mathrm{mg}$ RNA was reverse transcribed to cDNA. cDNA was amplified using the following primers: TLR4 forward, 5'-AAGTATGGCAGA GGTGAA-3' and reverse, 5'-GGAATAAAGTCCCTGTAG TG-3'; MyD88 forward, 5'-GCGATGTTTCCCACCCTT-3' and reverse, 5'-CTTCTTCCGCACGCTCAC-3'; GAP-43 forward, 5'-AGGAGGAGGGCAGCAAAG-3' and reverse, 5'-CGGCGAGTTATCAGTGGAAG; miR-204 forward, 5'-TGGCTACAGTCTTTCTTCA-3' and reverse, 5'-CTCATG GGACAGTTATGG-3'; NF-кB forward, 5'-CACCCTGAC CTTGCCTAT-3' and reverse, 5'-TGAAGCTGCCAGTGC TAT-3'; GAPDH forward, 5'-GTCTGCCACGATAACACC-3' and reverse, 5'-CAATACAACAAGCCCACTC-3'.
Western blot analysis. The retinal vascular tissue of the SD rats was homogenized in tissue lysis solution to disintegrate protein for $30 \mathrm{~min}$, following which the homogenates were centrifuged at $8,880 \mathrm{x} \mathrm{g}$, for $10 \mathrm{~min}$ at $4^{\circ} \mathrm{C}$. BCA method (Beyotime Institute of Biotechnology) was used to determine the protein concentrations. A total of $30 \mu$ protein was loaded for $10 \%$ SDS-PAGE and transferred onto immobilon P membranes (EMD Millipore, Billerica, MA, USA). The membranes were incubated with blocking solution of 5\% skim milk dissolved in Tris-buffered saline for $1 \mathrm{~h}$ at room temperature, followed by an overnight incubation with rabbit primary antibodies against GAP-43 (1:100,000, cat. no. ab75810), TLR4 (1:1,000, cat. no. ab22048), MyD88 (1:1,000, cat. no. ab2068)

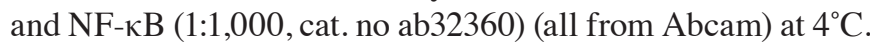
The membranes were next incubated with secondary horseradish peroxidase-conjugated donkey anti-rabbit $\operatorname{IgG}(1: 1,000$, cat. no. ab150077; Abcam) for $\sim 1 \mathrm{~h}$ at room temperature. Chemiluminescence was used to detect the relative levels of the target proteins, with $\beta$-actin used as an internal control. The blots were visualized by an enhanced chemiluminesence system (Amersham; GE Healtcare, Chicago, IL, USA).

TUNEL staining. As mentioned above, 20 healthy SD rats were randomly divided into four groups, each group containing 5 rats: Normal control group; model + miR-204 mimic group; model + miR-204 inhibitor group; and model group. In order to evaluate apoptosis in the retinas of the SD rats, a TUNEL assay was used for the detection of apoptotic cells. TUNEL-positive cells represent apoptotic cells (30). After injection of PBS $(100 \mathrm{ml})$ in the left ventricle of rats once every month for 8 months, the right atrial blood outflow was blocked, and tissues were fixed with $4 \%$ paraformaldehyde $(100 \mathrm{ml})$. On removal of the eye, it was completely stripped of the retina by trypsin digestion, following which the glass body and inner limiting membrane were peeled off, and retinal nerve tissue was removed. The complete retinal capillary network was transferred onto a slide with the retinal capillary network of the optic disc at the center, divided into three regions. The slide was examined in a blinded-manner under an optical microscope for counting cells and acellular vasculature. The retinal vasculature was then stained using the Dead end apoptosis detection system (Promega Corporation, Madison, WI, USA). TUNEL-positive cells were labeled with fluorescein-conjugated streptavidin and the numbers of TUNEL-positive cells were determined per unit area $\left(\mathrm{mm}^{2}\right)$.

Statistical analysis. Significant differences between groups were analyzed using one-way analysis of variance and Tukey's multiple comparison test in Prism 5.0 software (GraphPad Software, Inc., San Diego, CA, USA). P<0.05 was considered to indicate a statistically significant difference.

\section{Results}

Optic nerve injury upregulates miR-204 and inhibits the $m R N A$ expression of GAP-43. The levels of miR-204 and GAP-43 in the retina of the optic nerve injury model SD rats and the normal control SD rats were detected using RT-qPCR analysis, the results of which are shown in Fig. 1. The results showed that miR-204 was effectively induced by optic nerve 
A

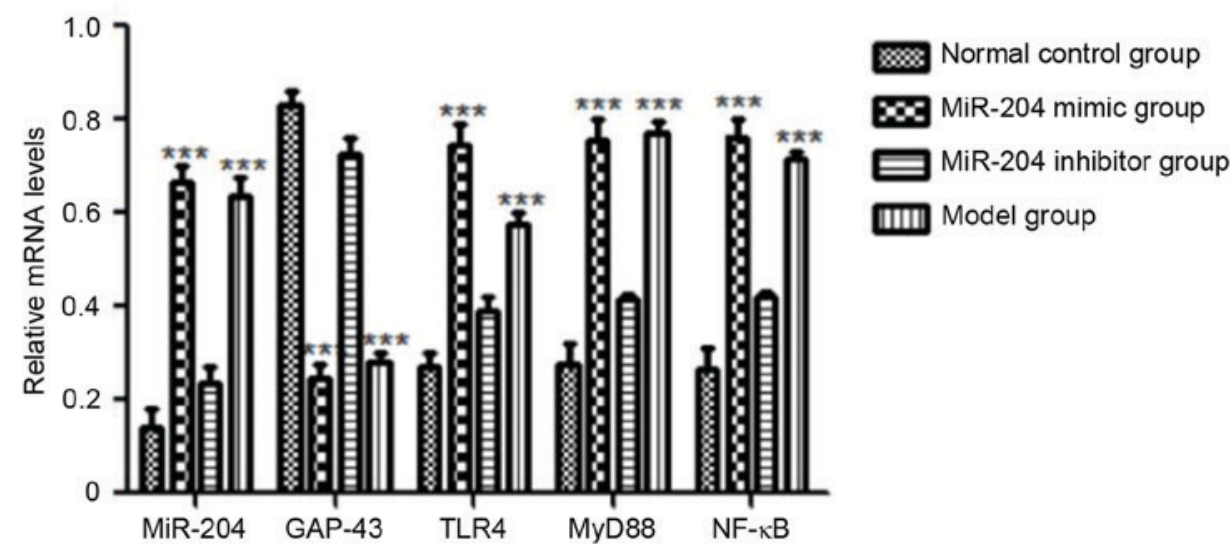

B

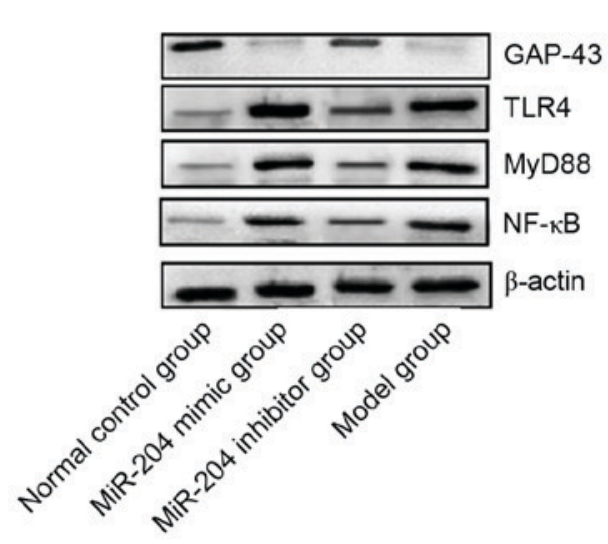

C

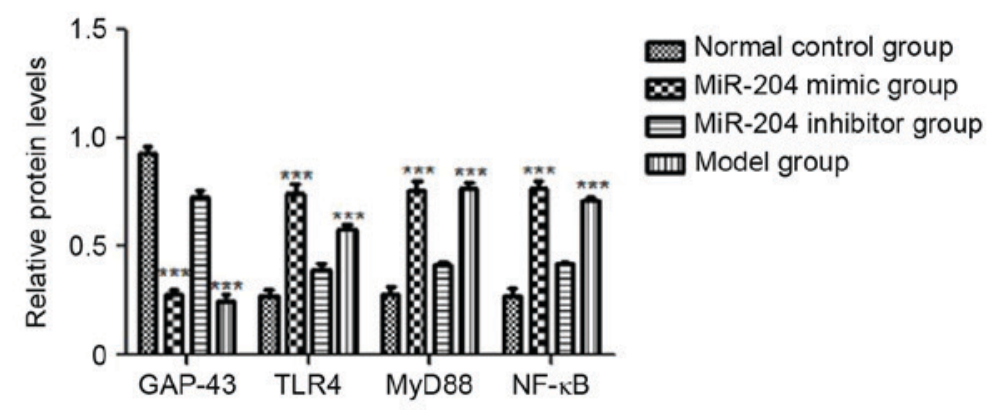

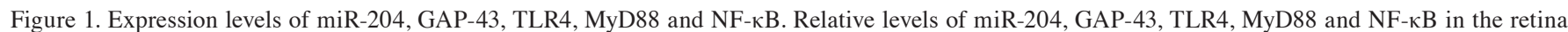
of each group demonstrated by (A) reverse transcription-quantitative polymerase chain reaction and (B) western blot analyses. (C) Quantification of protein levels. Compared with the normal SD rats, the level of miR-204 in SD rats with optic nerve injury was significantly increased $\left({ }^{* *} \mathrm{P}<0.05\right.$, $\left.{ }^{* * *} \mathrm{P}<0.01\right)$ and the mRNA level of GAP-43 was significantly inhibited by optic nerve injury $\left({ }^{* * *} \mathrm{P}<0.05,{ }^{* * * *} \mathrm{P}<0.01\right)$. miR-204 increased the gene levels of TLR4/MyD88/NF-kB, and decreased the mRNA and protein expression of GAP-43. miR, microRNA; GAP-43, growth-associated protein-43; TLR4, toll-like receptor 4; MyD88, myeloid

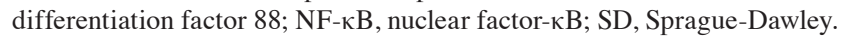

injury $(\mathrm{P}<0.05)$, whereas the mRNA level of neuroprotective GAP-43 was significantly inhibited by optic nerve injury $(\mathrm{P}<0.05)$, compared with that in the normal SD rats (Fig. 1A). This suggested that optic nerve injury was a destructive effect in the body.

miR-204 decreases the level of GAP-43 and promotes the TLR4/MyD88/NF- $\kappa B$ pathway. The levels of miR-204, GAP-43, TLR4, MyD88 and NF- $\kappa$ B in each group, following the overexpression and inhibition of miR-204 by injection of miR-204 mimic or miR-204 inhibitor, were detected using RT-qPCR analysis for miRNAs and mRNAs, and western blot analysis for proteins (Fig. 1A-C). As shown in Fig. 1A, which show the results of the RT-qPCR analysis of miR-204 and the GAP-43, TLR4, MyD88 and NF- $\kappa$ B mRNAs in each group, miR-204 was effectively increased by the miR-204 mimic, compared with that in the normal control group $(\mathrm{P}<0.05)$, which was the similar to the result in the model group (Fig. 1A). The results for GAP-43 were the opposite in these two groups, being significantly inhibited in the miR-204 mimic and model groups $(\mathrm{P}<0.05)$. Compared with the normal control group, the levels of TLR4, MyD88 and NF-кB in the miR-204 mimic group and model group were significantly increased, and the differences were statistically significant $(\mathrm{P}<0.05)$. These three genes were effectively inhibited by the miR-204 inhibitor, compared with model group (Fig. 1A). They remained marginally higher than those in the normal control group, however, the difference was not statistically significant. The proteins levels showed similar results to mRNAs for GAP-43, TLR4, MyD88 and NF- $\mathrm{KB}$ (Fig. 1B and C).

miR-204 increases the apoptosis of retinal cells. In order to evaluate apoptosis in the retina of SD rats, a TUNEL assay was used for the detection of apoptotic cells. TUNEL-positive cells represent the apoptotic cells, as shown in Fig. 2. The results showed that, compared with the normal control group, the apoptotic rates in the miR-204 mimic group and the model group were significantly increased (Fig. 2A and B). The numbers of TUNEL-positive cells in the retina of the miR-204 mimic group and the model group were significantly higher, compared with that of the control group $(\mathrm{P}<0.05)$. Compared with the normal control group, the number of TUNEL-positive cells of the miR-204 inhibitor group was increased, however, this difference was not statistically significant $(\mathrm{P}>0.05)$. 
A

Normal control

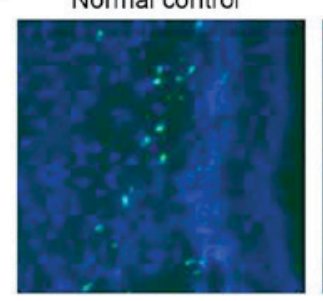

Mimic

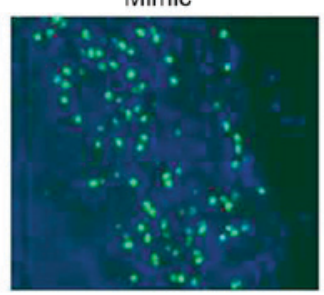

Inhibitor

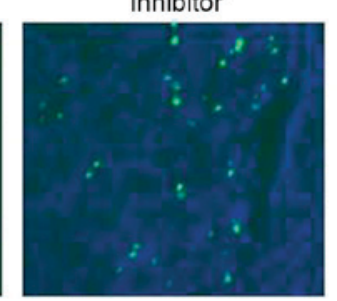

Model

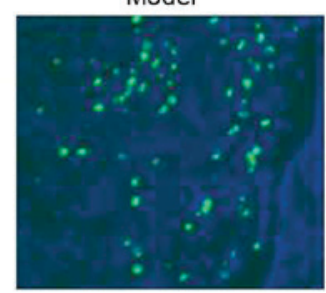

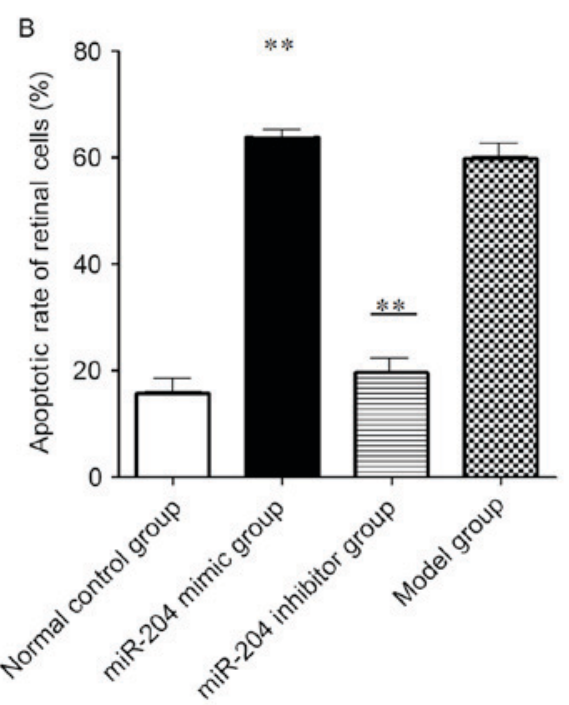

Figure 2. Apoptotic activity of retinal cells in each group. Apoptosis was analyzed using a TUNEL assay. (A) Positive TUNEL straining (green) was observed under a fluorescence microscope; magnification, x200. (B) Quantitative analysis. The number of apoptotic cells was calculated by averaging the number of positive TUNEL signals. Compared with the normal control group, there was a significant increase of TUNEL-positive cells in the miR-204 mimic and the model groups. ${ }^{* *} \mathrm{P}<0.05$, miR-204 mimic vs. normal control group and miR-204 inhibitor vs. model group. miR, microRNA; TUNEL, terminal deoxynucleotidyl transferase-mediated deoxyuridine triphosphate nick end labeling.

\section{Discussion}

The survival of RGCs is the most important requirement for regeneration of the optic nerve following injury, therefore, it is important to protect ganglion cell activity and inhibit the apoptosis of ganglion cells (31). GAP-43 is a presynaptic protein, which is key in the regulation of axonal growth and modulating synapse formation (6). The increase of GAP-43 can exert neuroprotective effects in the central nervous system, including the production of progesterone (7-9). It has been shown that the level of GAP-43 is enhanced in axon regeneration (32). However, the overexpression of GAP-43 was shown to aggravate RGC death in experimental chronic intraocular pressure (IOP) elevation injury, which indicates that the function of this same protein is complex according to the different pathogenesis (33). The results of the present study demonstrated that a low expression of GAP-43 (Fig. 1) was associated with the apoptotic activity of the retinal cell (Fig. 2). Optic nerve injury inhibited the level of GAP-43 (Fig. 1), which possibly reflected the destructive effect of optic nerve injury. Optic nerve injury activated the TLR4/MyD88/NF- $\mathrm{B}$ pathway (Fig. 1), which was consistent with the result of a previous study, which showed that neuroinflammation was involved in the death of RGCs following optic nerve injury (31).

The expression patterns of miRNAs have been analyzed in a variety of ocular tissues and multiple pathologies, and the downregulation of miRNAs, including miR-181c,
miR-497 and miR-204, have been associated with chronically elevated IOP (34). In addition, the significant elevation of miRNAs, including miR-223, miR142-5p and miR142-3p, may be involved in autoimmune uveoretinitis (35). miR-204 is a widely distributed miRNA, which has target genes belonging to different pathways, and is key in eye development and neural differentiation processes, including axon guidance (34). In addition, a key role of miR-204 in the differentiation of the RPE was verified in vivo through the RPE-specific conditional mutagenesis of Dicerl or Dger8 in mice (35). It has been found that miR-204 was expressed at high levels in nerve cells and axons (36). In the present study, the results showed that miR-204 was increased in the retinal injury model, which reflected adjusting of function to retinal damage repair.

When given sufficient light to stimulate rats, which have survived in the dark for 10 days, the expression level of GAP-43 has been shown to increase slowly, eventually reaching a maximum (37). This suggests that GAP-43 may be involved in the repair and regeneration of retinal light damage. A previous study found that, in the normal retina, the immunoreactivity of GAP-43 is predominantly in the inner plexiform layer, but in a rat model of retinal ischemia reperfusion injury model induced by elevated intraocular pressure, GAP-43 immunoreactivity appeared in retinal ganglion cells; the expression of GAP-43 peaked at $190 \%$ in 3 days and the rate reduced in 7 days (38). Therefore, it was hypothesized 
that optic ganglion cells have the potential for partial regeneration as GAP-43 level increases.

The present study observed the effects on the GAP-43 protein induced by miR-204 through the injection of miR-204 mimic and inhibitor in the established optic nerve injury model. GAP-43 was decreased in the miR-204 mimic group, whereas injection with miR-204 inhibitor increased the level of GAP-43 (Fig. 1). This suggested that miR-204 downregulated the protein expression of GAP-43, which was the similar to the result of a previous study, in which miR-204 was expressed at high levels in the glia, but low levels in the retinal neurons (23).

Studies have indicated that TLR4 is important in cerebral hemorrhage, cerebral infarction, and cerebral hemorrhage reperfusion injury (14-16). Another study indicated that TLR4 produces a response that causes brain damage (39). In the present study, retinal tissues were stretched using the technique of stretched preparation of the retina, following which the apoptosis of retinal cells was detected using the TUNEL method. The results showed that the apoptotic rates in the miR-204 mimic group and model group were significantly increased, compared with that in the normal control group $(\mathrm{P}<0.05)$. The apoptotic rate was effectively inhibited in the miR-204 inhibitor group, compared with that in the model group ( $\mathrm{P}<0.05$; Fig. 2). These results suggested that miR-204 was involved in retinal injury repair through modulating the expression of GAP-43.

The present study also examined the function of miR-204 in the TLR4, MyD88 and NF- $\kappa$ B pathways following injection of miR-204 mimic or inhibitor into the model rats using RT-qPCR and western blot analyses. The results showed that, compared with the normal control group, the levels of TLR4, MyD88 and NF- $\kappa$ B in the miR-204 mimic group and model group were significantly increased, and this difference was statistically significant $(\mathrm{P}<0.05)$. The miR-204 inhibitor effectively inhibited the expression levels of TLR4, MyD88 and $\mathrm{NF}-\kappa \mathrm{B}$, compared with those in the model group. The results showed that the levels of TLR4, MyD88 and NF- $\mathrm{BB}$ increased significantly in the optic nerve injury group, and treatment with miR-204 inhibitor following optic nerve injury decreased the levels of TLR4, MyD88 and NF- $\kappa \mathrm{B}$, which was consistent with the result of a previous study (13).

Taken together, the above results indicated that miR-204 may have been the result of the stress response of retinal injury, which downregulated the expression of neuroprotective factor GAP-43, and upregulated the levels of TLR4, MyD88 and $\mathrm{NF}-\kappa \mathrm{B}$. However, further experiments are required to elucidate the definite regulatory mechanism of miR-204 in retinal injury.

In conclusion, the results of the present study indicated that miR-204 promoted the apoptosis of retinal cells through inhibiting GAP-43, which provide theoretical guidance for the function of GAP-43 in retinal injury.

\section{Acknowledgements}

This study was supported by the Key Project of Jiangxi Science and Technology Agency: The Research and Application of The Standardized Diagnosis and Treatment of Diabetic Retinopathy in Province Model (grant no. 20161BBG70204).

\section{References}

1. Balla L, Ianovici N and Costin D: Pathology of the optic nerve injury. Rev Med Chir Soc Med Nat Iasi 116: 1087-1090, 2012.

2. Xue F, Wu K, Wang T, Cheng Y, Jiang M and Ji J: Morphological and functional changes of the optic nerve following traumatic optic nerve injuries in rabbits. Biomed Rep 4: 188-192, 2016.

3. Maxwell WL, Bartlett E and Morgan H: Wallerian degeneration in the optic nerve stretch-injury model of traumatic brain injury: A stereological analysis. J Neurotrauma 32: 780-790, 2015.

4. Grasselli G and Strata P: Structural plasticity of climbing fibers and the growth-associated protein GAP-43. Front Neural Circuits 7: 25, 2013.

5. Liu F, Liao F, Li W, Han Y and Liao D: Progesterone alters Nogo-A, GFAP and GAP-43 expression in a rat model of traumatic brain injury. Mol Med Rep 9: 1225-1231, 2014.

6. Zhao JC, Zhang LX, Zhang Y and Shen YF: The differential regulation of Gap43 gene in the neuronal differentiation of P19 cells. J Cell Physiol 227: 2645-2653, 2012.

7. Williams KR, McAninch DS, Stefanovic S, Xing L, Allen M, Li W, Feng Y, Mihailescu MR and Bassell GJ: hnRNP-Q1 represses nascent axon growth in cortical neurons by inhibiting Gap-43 mRNA translation. Mol Biol Cell 27: 518-534, 2016.

8. Zhu Q, Liu Z, Wang C, Nie L, He Y, Zhang Y, Liu X and Su G: Lentiviral-mediated growth-associated protein-43 modification of bone marrow mesenchymal stem cells improves traumatic optic neuropathy in rats. Mol Med Rep 12: 5691-5700, 2015.

9. Tan H, Kang X, Lu S and Liu L: The therapeutic effects of bone marrow mesenchymal stem cells after optic nerve damage in the adult rat. Clin Interv Aging 10: 487-490, 2015.

10. Kaneda M, Nagashima M, Mawatari K, Nunome T, Muramoto K, Sugitani K and Kato S: Growth-associated protein43 (GAP43) is a biochemical marker for the whole period of fish optic nerve regeneration. Adv Exp Med Biol 664: 97-104, 2010.

11. Guarnieri S, Morabito C, Paolini C, Boncompagni S, Pilla R, Fanò-Illic $\mathrm{G}$ and Mariggiò MA: Growth associated protein 43 is expressed in skeletal muscle fibers and is localized in proximity of mitochondria and calcium release units. PloS One 8: e53267, 2013.

12. Takei H, Buckleair LW, Rivera A and Powell SZ: Brain tissue microarrays in neurodegenerative diseases: Validation of methodology and immunohistochemical study of growth-associated protein-43 and calretinin. Pathol Int 57: 775-783, 2007.

13. Lucas SM, Rothwell NJ and Gibson RM: The role of inflammation in CNS injury and disease. Br J Pharmacol 147 (Suppl 1): S232-S240, 2006.

14. DeFrancesco-Lisowitz A, Lindborg JA, Niemi JP and Zigmond RE: The neuroimmunology of degeneration and regeneration in the peripheral nervous system. Neuroscience 302: 174-203, 2015.

15. Lin S, Liang Y, Zhang J, Bian C, Zhou H, Guo Q, Xiong Y, Li S and Su B: Microglial TIR-domain-containing adapter-inducing interferon- $\beta$ (TRIF) deficiency promotes retinal ganglion cell survival and axon regeneration via nuclear factor- $\kappa \mathrm{B}$. J Neuroinflammation 9: 39, 2012.

16. Qi M, Zheng L, Qi Y, Han X, Xu Y, Xu L, Yin L, Wang C, Zhao Y, Sun H, et al: Dioscin attenuates renal ischemia/reperfusion injury by inhibiting the TLR4/MyD88 signaling pathway via up-regulation of HSP70. Pharmacol Res 100: 341-352, 2015.

17. Chen M, Xiang $Z$ and Cai J: The anti-apoptotic and neuro-protective effects of human umbilical cord blood mesenchymal stem cells (hUCB-MSCs) on acute optic nerve injury is transient. Brain Res 1532: 63-75, 2013.

18. Latchney SE, Masiulis I, Zaccaria KJ, Lagace DC, Powell CM, McCasland JS and Eisch AJ: Developmental and adult GAP-43 deficiency in mice dynamically alters hippocampal neurogenesis and mossy fiber volume. Dev Neurosci 36: 44-63, 2014.

19. Bertoli G,Cava C and Castiglioni I: MicroRNAs: New biomarkers for diagnosis, prognosis, therapy prediction and therapeutic tools for breast cancer. Theranostics 5: 1122-1143, 2015.

20. Adlakha YK and Neeru Saini: Brain microRNAs and insights into biological functions and therapeutic potential of brain enriched miRNA-128. Mol Cancer 13: 33, 2014.

21. Ouyang YB, Stary CM, White RE and Giffard RG: The use of microRNAs to modialate redox and immune response to stroke. Antioxid Redox Signal 22: 187-202, 2015.

22. $\mathrm{Li} \mathrm{T}, \mathrm{Pan} \mathrm{H}$ and $\mathrm{Li} \mathrm{R}$ : The dual regulatory role of miR-204 in cancer. Tumor Biol 37: 11667-11677, 2016. 
23. Huang J, Zhao L, Xing L and Chen D: MicroRNA-204 regulates Runx 2 protein expression and mesenchymal progenitor cell differentiation. Stem Cells 28: 357-364, 2010.

24. Zhao J, Wang C, Song Y and Fang B: Arsenic trioxide and microRNA-204 display contrary effects on regulating adipogenic and osteogenic differentiation of mesenchymal stem cells in aplastic anemia. Acta Biochim Biophys Sin (Shanghai) 46: 885-893, 2014.

25. Banaei-Esfahani A, Moazzeni H, Naseri Nosar P, Amin S, Arefian E, Soleimani M, Yazdani S and Elahi E: MicroRNAs that target RGS5. Iran J Basic Med Sci 18: 108-114, 2015.

26. Ohana R, Weiman-Kelman B, Raviv S, Tamm ER, Pasmanik-Chor M, Rinon A, Netanely D, Shamir R, Solomon AS and Ashery-Padan R: MicroRNAs are essential for differentiation of the retinal pigmented epithelium and maturation of adjacent photoreceptors. Development 142: 2487-2498, 2015.

27. An J, Chen X, Chen W, Liang R, Reinach PS, Yan D and Tu L: MicroRNA expression profile and the role of miR-204 in corneal wound healing. Invest Ophthalmol Vis Sci 56: 3673-3683, 2015.

28. Natera-Naranjo O, Aschrafi A, Gioio AE and Kaplan BB: Identification and quantitative analyses of microRNAs located in the distal axons of sympathetic neurons. RNA 16: 1516-1529, 2010.

29. Livak KJ and Schmittgen TD: Analysis of relative gene expression data using real-time quantitative PCR and the 2(-Delta Delta C(T)) method. Methods 25: 402-408, 2001.

30. Kim J, Jo K, Lee IS, Kim CS and Kim JS: The extract of Aster koraiensis prevents retinal pericyte apoptosis in diabetic rats and its active compound, chlorogenic acid inhibits AGE formation and AGE/RAGE interaction. Nutrients 8: E585, 2016.

31. Morishita S, Oku H, Horie T, Tonari M, Kida T, Okubo A, Sugiyama T, Takai S, Hara H and Ikeda T: Systemic simvastatin rescues retinal ganglion cells from optic nerve injury possibly through suppression of astroglial NF-kB activation. PLoS One 9: e84387, 2014.
32. Gupta SK, Mishra R, Kusum S, Spedding M, Meiri KF, Gressens P and Mani S: GAP-43 is essential for the neurotrophic effects of BDNF and positive AMPA receptor modulator S18986. Cell Death Differ 16: 624-637, 2009.

33. Huang C, Cen LP, Liu L, Leaver SG, Harvey AR, Cui Q, Pang CP and Zhang M: Adeno-associated virus-mediated expression of growth-associated protein-43 aggravates retinal ganglion cell death in experimental chronic glaucomatous injury. Mol Vis 19: 1422-1432, 2013

34. Conte I, Merella S, Garcia-Manteiga JM, Migliore C, Lazarevic D, Carrella S, Marco-Ferreres R, Avellino R, Davidson NP and Emmett W: The combination of transcriptomics and informatics identifies pathways targeted by miR-204 during neurogenesis and axon guidance. Nucleic Acids Res 42: 7793-7806, 2014.

35. Ohana R, Weiman-Kelman B, Raviv S, Tamm ER, Pasmanik-Chor M, Rinon A, Netanely D, Shamir R, Solomon AS and Ashery-Padan R: MicroRNAs are essential for differentiation of the retinal pigmented epithelium and maturation of adjacent photoreceptors. Development 142: 2487-2498, 2015.

36. Natera-Naranjo O, Aschrafi A, Gioio AE and Kaplan BB: Identification and quantitative analyses of microRNAs located in the distal axons of sympathetic neurons. RNA 16: 1516-1529, 2010.

37. López-Costa JJ, Goldstein J, Mangeaud M and Saavedra JP: Expression of GAP-43 in the retina of rats following protracted illumination. Brain Res 900: 332-336, 2001.

38. Ju WK, Gwon JS, Park SJ, Kim KY, Moon JI, Lee MY, Oh SJ and Chun MH: Growth-associated protein 43 is up-regulated in the ganglion cells of the ischemic rat retina. Neuroreport 13: $861-865,2002$

39. Alfonso-Loeches S, Pascual-Lucas M, Blanco AM Sanchez-Vera I and Guerri C: Pivotal role of TLR4 receptors in alcohol-induced neuroinflammation and brain damage. J Neurosci 30: 8285-8295, 2010. 